\title{
ON THE DIFFERENTIAL OPERATORS OF THE GENERALIZED FIFTH-ORDER KORTEWEG-DE VRIES EQUATION*
}

\author{
CHUN-TE LEE ${ }^{\dagger}$
}

\begin{abstract}
In this paper, we present the differential operators of the generalized fifth-order KdV equation. We give formal proofs on the Hamiltonian property including the skew-adjoint property and Jacobi identity by the use of prolongation method. Our results show that there are five 3-order Hamiltonian operators, which can be used to construct the Hamiltonians, and no 5-order operators are shown to pass the Hamiltonian test, although there are infinite number of them, and are skewadjoint.
\end{abstract}

Key words. Hamiltonian system, Nonlinear differential equation, Nonlinear partial differential equation, Fifth-order KdV equation, Ito equation, Sawada-Kotera equation, Caudrey-Dodd-Gibbon equation, Kaup-Kupershmidt equation, Lax equation, Jacobi identity, skew-adjoint operator, prolongation.

AMS subject classifications. 37K10, 37K05, 35Q53, 35G20, 35L05, 47J35

1. Introduction. The study of the generalized fifth-order Korteweg-de Vries (fifth-order KdV) equation

$$
u_{t}+\alpha u u_{x x x}+\beta u_{x} u_{x x}+\gamma u^{2} u_{x}+u_{x x x x x}=0,
$$

where $\alpha, \beta$ and $\gamma$ are arbitrary real parameters, has always been an important topic in nonlinear physical phenomena. This equation not only describes the motions of long waves in shallow water under gravity and in a one-dimensional nonlinear lattice but also is an important mathematical model with wide applications in quantum mechanics and nonlinear optics. Typical examples are widely used in various fields such as solid state physics, plasma physics, fluid physics and quantum field theory. A great deal of research work has been conducted during the past decades for exact solutions, such as soliton solutions [1, 2, 3, 4]. Several different approaches, such as Backlund transformation, a bilinear form, and Lax pairs, have been used independently for some constants $\alpha, \beta$ and $\gamma$, by which some of the soliton and multi-soliton solutions are obtained [5]. Interesting and deeply examined examples of the fifth-order $\mathrm{KdV}$ equation are

- Sawada-Kotera equation (SK equation) [6]

$$
u_{t}+5 u u_{x x x}+5 u_{x} u_{x x}+5 u^{2} u_{x}+u_{x x x x x}=0 .
$$

- Caudrey-Dodd-Gibbon equation (CDG equation) [7]

$$
u_{t}+30 u u_{x x x}+30 u_{x} u_{x x}+180 u^{2} u_{x}+u_{x x x x x}=0 .
$$

- Lax equation [8]

$$
u_{t}+10 u u_{x x x}+20 u_{x} u_{x x}+30 u^{2} u_{x}+u_{x x x x x}=0 .
$$

- Kaup-Kupershmidt-type equation I (KK I equation, [9])

$$
u_{t}+10 u u_{x x x}+25 u_{x} u_{x x}+20 u^{2} u_{x}+u_{x x x x x}=0 .
$$

\footnotetext{
*Received January 12, 2010; accepted for publication June 4, 2010.

${ }^{\dagger}$ Mathematical Institute, Univerisity of Oxford, 24-29 St. Giles', Oxford, OX1 3LB, UK (chuntelee2000@googlemail.com). PACS no. 02.30.Jr, 05.45.Yv, 02.30.Gp, 02.30.Ik, 02.30.Tb.
} 
- Kaup-Kupershmidt-type equation II (KK II equation, [10, 11])

$$
u_{t}-15 u u_{x x x}-\frac{75}{2} u_{x} u_{x x}+45 u^{2} u_{x}+u_{x x x x x}=0 .
$$

- Ito equation [12]

$$
u_{t}+3 u u_{x x x}+6 u_{x} u_{x x}+2 u^{2} u_{x}+u_{x x x x x}=0 .
$$

As the constants $\alpha, \beta$ and $\gamma$ taking different values, the properties of Eq. (1) drastically change. For instance, the Lax equation with $\alpha=10, \beta=20$, and $\gamma=30$ and SK equation where $\alpha=\beta=\gamma=5$ are completely integrable and have $N$-soliton solutions [6]. The KK-type equations (KK I, KKII) with $\alpha=10, \beta=25, \gamma=20$ and $\alpha=-15, \beta=-\frac{75}{2}, \gamma=45$ respectively are known to be integrable too, and has bilinear representations $[10,11,13]$. The Ito equation with $\alpha=3, \beta=6$, and $\gamma=2$ is not integrable, but has a limited number of special conserved densities [14]. We also note that the Caudrey-Dodd-Gibbon equation can be transformed to the Sawada-Kotera equation through a particular scaling transformation for $u$. So they are the same equation.

As far as we are concerned, the question of Hamiltonian structures for the fifthorder KdV equation is very important and deserves serious considerations, since soliton equations often come with some surprising Hamiltonian structures and are now being recognized as an important aspect in soliton theory [15, 16, 17].

In this paper, we rummage through all possible differential operators for the generalized fifth-order KdV equation (1), and give full validation tests of their Hamiltonian structures regarding the skew-adjoint property and the Jacobi identity. Such two important properties will be verified in a systematic way carefully and directly through prolongation method, and we will present all possible Hamiltonian operators for equation (1).

2. Preliminaries. Most integrable systems are known to have Hamiltonian structures. These are systems whose dynamical equations can be described through Hamilton's equations. The general form of an infinite-dimensional Hamiltonian system takes the form

$$
\frac{\partial u}{\partial t}+\mathcal{D} \frac{\delta \mathcal{H}}{\delta u}=0
$$

where $\mathcal{D}$ is a skew-adjoint operator and $\mathcal{H}$ is a functional

$$
\mathcal{H}\left[u, u^{(n)}\right]:=\int_{-\infty}^{\infty} H\left(u, u_{x}, \ldots, u_{n x}\right) \mathrm{d} x
$$

with the density function $H$ coming from the space of differentiable functions. Then one can define the Poisson bracket as

$$
\{\mathcal{F}, \mathcal{G}\}=\int_{-\infty}^{\infty} \frac{\delta \mathcal{F}}{\delta u} \mathcal{D} \frac{\delta \mathcal{G}}{\delta u} \mathrm{~d} x
$$

for any two smooth functionals $\mathcal{F}, \mathcal{G}$. For instance, it is well known that the $\mathrm{KdV}$ equation

$$
u_{t}-6 u u_{x}+u_{x x x}=0
$$


admits a Hamiltonian system as

$$
\frac{\partial u}{\partial t}=\mathcal{D} \frac{\delta \mathcal{H}}{\delta u}, \quad \mathcal{H}=\int\left(\frac{1}{2} u_{x}^{2}+u^{3}\right) \mathrm{d} x
$$

where $\mathcal{D}=\partial_{x}, \mathcal{H}$ is a functional called Hamiltonian and $\delta / \delta u$ is the variational derivative. Its Poisson structure is

$$
\{\mathcal{F}, \mathcal{G}\}=\int_{-\infty}^{\infty} \frac{\delta \mathcal{F}}{\delta u} \partial_{x} \frac{\delta \mathcal{G}}{\delta u} \mathrm{~d} x
$$

A general differential operator $\mathcal{D}$ is of the form

$$
\mathcal{D}:=\sum_{i=0}^{n} P_{i}\left(u, u_{x}, \ldots, u_{n x}\right) \frac{\partial^{i}}{\partial x^{i}}
$$

for some smooth differentiable functions $P_{i}$, where $n$ is a finite, natural number. For simplicity, one can write $\mathcal{D}$ as

$$
\mathcal{D}=\sum_{J} P_{J} \partial_{J}
$$

where $J=1,2, \ldots, n$, for some finite number $n$, and $\partial_{J}:=\partial^{J} / \partial x^{J}$. A differential operator $\mathcal{D}$ is of order $n$ if its leading coefficient is not zero, i.e. $P_{n} \neq 0$, and of order 0 if it is a single differentiable function.

Definition 1. Let $\mathcal{A}$ be the space of differentiable functions, suppose

$$
\mathcal{D}=\sum_{J} P_{J} \partial_{J}, \quad P_{j} \in \mathcal{A},
$$

then its adjoint $\mathcal{D}^{*}$ is a differential operator which satisfies

$$
\int P \mathcal{D} Q \mathrm{~d} x=\int Q \mathcal{D}^{*} P \mathrm{~d} x, \quad \forall P, Q \in \mathcal{A} .
$$

COROLlaRY 1. The adjoint of a differential operator can be written as

$$
\mathcal{D}^{*}=\sum_{J}\left(-\partial_{J}\right) \cdot P_{J} \cdot
$$

which means that for any $Q \in \mathcal{A}$, we have

$$
\mathcal{D}^{*} Q=\sum_{J}\left(-\partial_{J}\right) \cdot\left[P_{J} Q\right] .
$$

Moreover, an operator $\mathcal{D}$ is self-adjoint if $\mathcal{D}^{*}=\mathcal{D}$, and skew-adjoint if $\mathcal{D}^{*}=-\mathcal{D}$.

Definition 2. A differential operator $\mathcal{D}$ is called Hamiltonian if its Poisson bracket (10) satisfies the "skew-symmetry" property

$$
\{\mathcal{P}, \mathcal{Q}\}=-\{\mathcal{Q}, \mathcal{P}\}
$$


and the "Jacobi identity"

$$
\{\mathcal{P},\{\mathcal{Q}, \mathcal{R}\}\}+\{\{\mathcal{R}, \mathcal{P}\}, \mathcal{Q}\}+\{\{\mathcal{Q}, \mathcal{R}\}, \mathcal{P}\}=0,
$$

for any smooth functionals $\mathcal{P}, \mathcal{Q}$, and $\mathcal{R}$.

We note that if $\mathcal{D}$ is a skew-adjoint differential operator whose coefficients do not depend on $u$ or its derivatives, then $\mathcal{D}$ is automatically a Hamiltonian operator.

Although we should check the Jacobi identity in (18) by direct verifications, it still requires quite a lot of calculations even in such a relatively simple example like the KdV equation. A radical simplification is offered by Olver [18] using the theory of multi-vectors and prolongation of the vector field. In this section we will apply such method to justify a Hamiltonian operator.

Let $\mathcal{A}^{q}$ be the space of general $q$-tuple differentiable functions. One can define the general multi $k$-vector as follows

$$
\Theta=\int\left(\sum_{\alpha, J} R_{J}^{\alpha}\left(u(x), u^{(n)}(x)\right) \theta_{J_{1}}^{\alpha_{1}} \wedge \cdots \wedge \theta_{J_{k}}^{\alpha_{k}}\right) \mathrm{d} x,
$$

where $R_{J}^{\alpha} \in \mathcal{A}^{q}$ are differentiable functions depending on $u$ and derivatives of $u$ up to some finite number. Here $x=\left(x^{1}, \ldots, x^{p}\right)$ are independent variables, $u=\left(u^{1}, \ldots, u^{q}\right)$ are dependent variables and $J=\left(J_{1}, J_{2}, \ldots, J_{k}\right)$ is a $k$-th order multi-index with $0 \leq J_{k} \leq p$ indicating which derivatives are being taken. Here $\alpha=\left(\alpha_{1}, \alpha_{2}, \ldots, \alpha_{n}\right)$ is a multi-index with $1 \leq \alpha_{i} \leq q$ indicating which variable are being using. The variables $\theta_{J i}^{\alpha_{i}}$ are unit vectors of $u$ corresponding to the derivatives $\partial / \partial u_{J_{i}}^{\alpha_{i}}$ and $\wedge$ is the wedge product. Olver [18] further indicated that any skew-adjoint differential operator $\mathcal{D}$ can be written as a canonical form of functional bi-vector

$$
\Theta_{\mathcal{D}}=\frac{1}{2} \int\{\theta \wedge \mathcal{D} \theta\} \mathrm{d} x=\frac{1}{2} \int\left(\sum_{\alpha, \beta=1}^{q} \theta^{\alpha} \wedge \mathcal{D}_{\alpha \beta} \theta^{\beta}\right) \mathrm{d} x
$$

where $\mathcal{D}=\left(\mathcal{D}_{\alpha \beta}\right)$ is a $q \times q$-dimensional differential operator. Thus studying the Hamiltonian operator of the differential equation is equally important as studying the bi-vector of the equation.

ThEOREM 1. Let $\mathcal{D}$ be a skew-adjoint operator and let $\theta$ be a unit vector of $\mathcal{D}$, suppose

$$
\Theta_{\mathcal{D}}=\frac{1}{2} \int(\theta \wedge \mathcal{D}(\theta)) \mathrm{d} x
$$

is the corresponding functional bi-vector. Then $\mathcal{D}$ is Hamiltonian if and only if

$$
\operatorname{pr} \mathbf{v}_{\mathcal{D} \theta}\left(\Theta_{\mathcal{D}}\right)=0
$$

where "pr" stands for prolongation calculations.

3. Three-order operator. We start with the general three-order differential operator of the form

$$
\mathcal{D}=\partial_{x}^{3}+\alpha_{1} u \partial_{x}+\beta_{1} u_{x}
$$


where $\alpha_{1}$ and $\beta_{1}$ are real parameters to be determined. In order to make the fifth-order $\mathrm{KdV}$ equation (1) from (8), we take a guess at the Hamiltonian form as

$$
\mathcal{H}=\int\left(\frac{1}{2} u u_{x x}+\frac{\gamma_{1}}{3} u^{3}\right) \mathrm{d} x
$$

where $\gamma_{1}$ is a real parameter to be determined too. Then one can substitute (23) and (24) into (8) to get

$$
u_{t}+\left(\partial_{x}^{3}+\alpha_{1} u \partial_{x}+\beta_{1} u_{x}\right)\left(u_{x x}+\gamma_{1} u^{2}\right)=0
$$

and we obtain a nonlinear system of algebraic equations for $\alpha_{1}, \beta_{1}$ and $\gamma_{1}$ :

(1) SK equation :

$$
\left\{\begin{array}{l}
2 \gamma_{1}+\alpha_{1}=5, \\
6 \gamma_{1}+\beta_{1}=5, \\
2 \alpha_{1} \gamma_{1}+\beta_{1} \gamma_{1}=5
\end{array}\right.
$$

Then we have solution $\alpha_{1}=3, \beta_{1}=-1$ and $\gamma_{1}=1$, and the "potential" Hamiltonian operator as well as the Hamiltonian are

$$
\begin{aligned}
\mathcal{D}_{s k-3} & =\partial_{x}^{3}+3 u \partial_{x}-u_{x}, \\
\mathcal{H}_{s k-3} & =\int\left(\frac{1}{2} u u_{x x}+\frac{1}{3} u^{3}\right) \mathrm{d} x .
\end{aligned}
$$

(2) CDG equation :

$$
\left\{\begin{array}{l}
2 \gamma_{1}+\alpha_{1}=30 \\
6 \gamma_{1}+\beta_{1}=30 \\
2 \alpha_{1} \gamma_{1}+\beta_{1} \gamma_{1}=180
\end{array}\right.
$$

Then we have $\alpha_{1}=18, \beta_{1}=-6$ and $\gamma_{1}=6$, and the "potential" Hamiltonian operator as well as the Hamiltonian are

$$
\begin{aligned}
& \mathcal{D}_{C D G-3}=\partial_{x}^{3}+18 u \partial_{x}-6 u_{x} \\
& \mathcal{H}_{C D G-3}=\int\left(\frac{1}{2} u u_{x x}+2 u^{3}\right) \mathrm{d} x .
\end{aligned}
$$

(3) Lax equation :

$$
\left\{\begin{array}{l}
2 \gamma_{1}+\alpha_{1}=10, \\
6 \gamma_{1}+\beta_{1}=20 \\
2 \alpha_{1} \gamma_{1}+\beta_{1} \gamma_{1}=30
\end{array}\right.
$$

Then we have $\alpha_{1}=4, \beta_{1}=2$ and $\gamma_{1}=3$, and the "potential" Hamiltonian operator as well as the Hamiltonian are

$$
\begin{aligned}
\mathcal{D}_{\text {Lax }-3} & =\partial_{x}^{3}+4 u \partial_{x}+2 u_{x}, \\
\mathcal{H}_{\text {Lax }-3} & =\int\left(\frac{1}{2} u u_{x x}+u^{3}\right) \mathrm{d} x .
\end{aligned}
$$


(4) KK I equation :

$$
\left\{\begin{array}{l}
2 \gamma_{1}+\alpha_{1}=10, \\
6 \gamma_{1}+\beta_{1}=25, \\
2 \alpha_{1} \gamma_{1}+\beta_{1} \gamma_{1}=20 .
\end{array}\right.
$$

Then we have $\alpha_{1}=2, \beta_{1}=1$ and $\gamma_{1}=4$, and the "potential" Hamiltonian operator as well as the Hamiltonian are

$$
\begin{aligned}
& \mathcal{D}_{K K I-3}=\partial_{x}^{3}+2 u \partial_{x}+u_{x}, \\
& \mathcal{H}_{K K I-3}=\int\left(\frac{1}{2} u u_{x x}+\frac{4}{3} u^{3}\right) \mathrm{d} x .
\end{aligned}
$$

(5) KK II equation:

$$
\left\{\begin{array}{l}
2 \gamma_{1}+\alpha_{1}=-15, \\
6 \gamma_{1}+\beta_{1}=-\frac{75}{2}, \\
2 \alpha_{1} \gamma_{1}+\beta_{1} \gamma_{1}=45
\end{array}\right.
$$

Then we have $\alpha_{1}=-3, \beta_{1}=-\frac{3}{2}$ and $\gamma_{1}=-6$, and the "potential" Hamiltonian operator as well as the Hamiltonian are

$$
\begin{aligned}
& \mathcal{D}_{K K I I-3}=\partial_{x}^{3}-3 u \partial_{x}-\frac{3}{2} u_{x}, \\
& \mathcal{H}_{K K I I-3}=\int\left(\frac{1}{2} u u_{x x}-2 u^{3}\right) \mathrm{d} x .
\end{aligned}
$$

(6) Ito equation :

$$
\left\{\begin{array}{l}
2 \gamma_{1}+\alpha_{1}=3, \\
6 \gamma_{1}+\beta_{1}=6, \\
2 \alpha_{1} \gamma_{1}+\beta_{1} \gamma_{1}=2 .
\end{array}\right.
$$

Then we have $\alpha_{1}=1, \beta_{1}=0$ and $\gamma_{1}=1$, and the "potential" Hamiltonian operator as well as the Hamiltonian are

$$
\begin{aligned}
\mathcal{D}_{\text {Ito- }-3} & =\partial_{x}^{3}+u \partial_{x}, \\
\mathcal{H}_{\text {Ito- }-3} & =\int\left(\frac{1}{2} u u_{x x}+\frac{1}{3} u^{3}\right) \mathrm{d} x .
\end{aligned}
$$

4. Three-order operator revisited. In this section we focus on 3-order, skewadjoint differential operator form as

$$
\mathcal{D}=\partial_{x}^{3}+2 a \partial_{x}+a_{x}
$$

where $a=a(x, t)$ is an analytic function to be determined.

Now technically we set $a(x, t)=\alpha_{2} u(x, t)$, where $\alpha_{2}$ is a real parameter to be determined. A quick judgement of (1), (8) and (37) gives the Hamiltonian form

$$
\mathcal{H}=\int\left(\frac{\beta_{2}}{2} u u_{x x}+\frac{\gamma_{2}}{3} u^{3}\right) \mathrm{d} x,
$$

where $\beta_{2}, \gamma_{2}$ are real parameters to be determined. We substitute (37) and (38) into (8) to get

$$
u_{t}+\left(\partial_{x}^{3}+2 a \partial_{x}+a_{x}\right)\left(\beta_{2} u_{x x}+\gamma_{2} u^{2}\right)=0,
$$

and obtain the following nonlinear system of algebraic equations for $\alpha_{2}, \beta_{2}$ and $\gamma_{2}$ : 
(1) SK equation :

$$
\left\{\begin{array}{l}
6 \gamma_{2}+\alpha_{2} \beta_{2}=5 \\
2 \gamma_{2}+2 \alpha_{2} \beta_{2}=5 \\
5 \alpha_{2} \gamma_{2}=5
\end{array}\right.
$$

Solving the above nonlinear system, we get $\alpha_{2}=2, \beta_{2}=1$ and $\gamma_{2}=1 / 2$, and

$$
\begin{aligned}
\mathcal{D}_{s k-3-1} & =\partial_{x}^{3}+4 u \partial_{x}+2 u_{x}, \\
\mathcal{H}_{s k-3-1} & =\int\left(\frac{1}{2} u u_{x x}+\frac{1}{6} u^{3}\right) \mathrm{d} x .
\end{aligned}
$$

(2) CDG equation :

$$
\left\{\begin{array}{l}
6 \gamma_{2}+\alpha_{2} \beta_{2}=30 \\
2 \gamma_{2}+2 \alpha_{2} \beta_{2}=30 \\
5 \alpha_{2} \gamma_{2}=180
\end{array}\right.
$$

Solving the above nonlinear system, we have $\alpha_{2}=12, \beta_{2}=1$ and $\gamma_{2}=3$, and

$$
\begin{aligned}
\mathcal{D}_{C D G-3-1} & =\partial_{x}^{3}+24 u \partial_{x}+12 u_{x}, \\
\mathcal{H}_{C D G-3-1} & =\int\left(\frac{1}{2} u u_{x x}+u^{3}\right) \mathrm{d} x .
\end{aligned}
$$

(3) Lax equation :

$$
\left\{\begin{array}{l}
6 \gamma_{2}+\alpha_{2} \beta_{2}=20 \\
2 \gamma_{2}+2 \alpha_{2} \beta_{2}=10 \\
5 \alpha_{2} \gamma_{2}=30
\end{array}\right.
$$

The above system admits $\alpha_{2}=4, \beta_{2}=1$ and $\gamma_{2}=3$, so that we have

$$
\begin{aligned}
& \mathcal{D}_{\text {Lax-3-1 }}=\partial_{x}^{3}+4 u \partial_{x}+2 u_{x}, \\
& \mathcal{H}_{\text {Lax-3-1 }}=\int\left(\frac{1}{2} u u_{x x}+u^{3}\right) \mathrm{d} x .
\end{aligned}
$$

We note that this is the same as $\mathcal{D}_{\text {Lax }-3}$.

(4) KK I equation :

$$
\left\{\begin{array}{l}
6 \gamma_{2}+\alpha_{2} \beta_{2}=25 \\
2 \gamma_{2}+2 \alpha_{2} \beta_{2}=10 \\
5 \alpha_{2} \gamma_{2}=20
\end{array}\right.
$$

Then the above system gives $\alpha_{2}=1, \beta_{2}=1$ and $\gamma_{2}=4$, and hence

$$
\begin{aligned}
\mathcal{D}_{K K I-3-1} & =\partial_{x}^{3}+2 u \partial_{x}+u_{x}, \\
\mathcal{H}_{K K I I-3-1} & =\int\left(\frac{1}{2} u u_{x x}+\frac{4}{3} u^{3}\right) \mathrm{d} x .
\end{aligned}
$$

We note that this is the same as $\mathcal{D}_{K K I-3}$. 
(5) KK II equation :

$$
\left\{\begin{array}{l}
6 \gamma_{2}+\alpha_{2} \beta_{2}=-\frac{75}{2} \\
2 \gamma_{2}+2 \alpha_{2} \beta_{2}=-15 \\
5 \alpha_{2} \gamma_{2}=45
\end{array}\right.
$$

The above system gives $\alpha_{2}=-3 / 2, \beta_{2}=1$ and $\gamma_{2}=-6$, so that we have

$$
\begin{aligned}
& \mathcal{D}_{K K I I-3-1}=\partial_{x}^{3}-3 u \partial_{x}-\frac{3}{2} u_{x}, \\
& \mathcal{H}_{K K I I-3-1}=\int\left(\frac{1}{2} u u_{x x}-2 u^{3}\right) \mathrm{d} x .
\end{aligned}
$$

We note that this is the same as $\mathcal{D}_{K K I I-3}$.

(6) Ito equation :

$$
\left\{\begin{array}{l}
6 \gamma_{2}+\alpha_{2} \beta_{2}=6 \\
2 \gamma_{2}+2 \alpha_{2} \beta_{2}=3 \\
5 \alpha_{2} \gamma_{2}=2
\end{array}\right.
$$

The above system is a contradictory linear system of equations, and no solutions for $\alpha_{2}, \beta_{2}$ and $\gamma_{2}$ can be found.

5. Fifth-order operator. In this section take the fifth-order, skew-adjoint operator of the form

$$
\mathcal{D}=\partial_{x}^{5}+a \partial_{x}^{3}+\partial_{x}^{3} a+b \partial_{x}+\partial_{x} b
$$

where $a=a(x, t), b=b(x, t)$ are to be determined. We suppose that

$$
a=\alpha_{3} u, \quad b=\beta_{3} u_{x x}+\gamma_{3} u^{2},
$$

where $\alpha_{3}, \beta_{3}$ and $\gamma_{3}$ are real parameters to be determined. A quick judgement of suitable Hamiltonian form from (8) and (49) is

$$
\mathcal{H}=\int \frac{1}{2} u^{2} \mathrm{~d} x
$$

Substituting (49) and (51) into (8), we obtain

$$
u_{t}+\left[\begin{array}{c}
u_{x x x x x}+2 \alpha_{3} u u_{x x x}+\alpha_{3} u_{x x x} u+3 \alpha_{3} u_{x x} u_{x}+3 \alpha_{3} u_{x} u_{x x} \\
+2\left(\beta_{3} u_{x x}+\gamma_{3} u^{2}\right) u_{x}+\left(\beta_{3} u_{x x x}+2 \gamma_{3} u u_{x}\right) u
\end{array}\right]=0
$$

and nonlinear system of algebraic equations for $\alpha_{3}, \beta_{3}$ and $\gamma_{3}$ :

(1) SK equation :

$$
\left\{\begin{array}{l}
3 \alpha_{3}+\beta_{3}=5 \\
6 \alpha_{3}+2 \beta_{3}=5 \\
4 \gamma_{3}=5
\end{array}\right.
$$

This system is a contradictory system of linear equations with no solutions can be found. 
(2) CDG equation :

$$
\left\{\begin{array}{l}
3 \alpha_{3}+\beta_{3}=30, \\
6 \alpha_{3}+2 \beta_{3}=30, \\
4 \gamma_{3}=180 .
\end{array}\right.
$$

This system is a contradictory system of linear equations with no solutions can be found.

(3) Lax equation :

$$
\left\{\begin{array}{l}
3 \alpha_{3}+\beta_{3}=10 \\
6 \alpha_{3}+2 \beta_{3}=20, \\
4 \gamma_{3}=30
\end{array}\right.
$$

This system gives infinite solutions under the condition $3 \alpha_{3}+\beta_{3}=10$ and $\gamma_{3}=15 / 2$. It gives infinite numbers of skew-adjoint operators as

$$
\begin{aligned}
\mathcal{D}_{\text {Lax }-5} & =\left(\begin{array}{c}
\partial_{x}^{5}+2 \alpha_{3} u \partial_{x x x}+\left(\alpha_{3}+\beta_{3}\right) u_{x x x} \\
+\left(3 \alpha_{3}+2 \beta_{3}\right) u_{x x} \partial_{x}+3 \alpha_{3} u_{x} \partial_{x x} \\
+15 u^{2} \partial_{x}+15 u u_{x}
\end{array}\right), \\
\mathcal{H}_{\text {Lax }-5} & =\int \frac{1}{2} u^{2} \mathrm{~d} x
\end{aligned}
$$

(4) KK I equation :

$$
\left\{\begin{array}{l}
3 \alpha_{3}+\beta_{3}=10, \\
6 \alpha_{3}+2 \beta_{3}=25, \\
4 \gamma_{3}=20
\end{array}\right.
$$

This system is a contradictory system, meaning no solutions can be found for $\alpha_{3}, \beta_{3}$ and $\gamma_{3}$.

(5) KK II equation :

$$
\left\{\begin{array}{l}
3 \alpha_{3}+\beta_{3}=-15 \\
6 \alpha_{3}+2 \beta_{3}=-\frac{75}{2}, \\
4 \gamma_{3}=45
\end{array}\right.
$$

This system contains contradictory linear equations such that no solutions can be found for suitable $\alpha_{3}, \beta_{3}$ and $\gamma_{3}$.

(6) Ito equation :

$$
\left\{\begin{array}{l}
3 \alpha_{3}+\beta_{3}=3, \\
6 \alpha_{3}+2 \beta_{3}=6, \\
4 \gamma_{3}=2
\end{array}\right.
$$

This system gives solutions when $3 \alpha_{3}+\beta_{3}=3$ and $\gamma_{3}=1 / 2$, so that we have

$$
\begin{aligned}
\mathcal{D}_{\text {Ito }-5} & =\left(\begin{array}{c}
\partial_{x}^{5}+2 \alpha_{3} u \partial_{x x x}+\left(\alpha_{3}+\beta_{3}\right) u_{x x x} \\
+\left(3 \alpha_{3}+2 \beta_{3}\right) u_{x x} \partial_{x}+3 \alpha_{3} u_{x} \partial_{x x} \\
+u^{2} \partial_{x}+u u_{x}
\end{array}\right), \\
\mathcal{H}_{\text {Ito }-5} & =\int \frac{1}{2} u^{2} \mathrm{~d} x .
\end{aligned}
$$


6. Classification of the differential operators. In previous sections, we apply the operator multiplications to obtain many "potential" Hamiltonian operators of 3-order and 5-order for the fifth-order KdV equations (1). These operators are all nontrivial, but need to be further verified for its Hamiltonian nature. In this section we would use the method of prolongation [18] to verify the skew-adjoint property and Jacobi identity in order to justify the Hamiltonian structure for the equation (1).

6.1. Skew and non-skew adjoint operator. First we note that

$$
\begin{aligned}
\mathcal{D}_{s k-3}^{*} & =-\partial_{x}^{3}-\partial_{x} \cdot(3 u)-u_{x} \\
& =-\partial_{x}^{3}-3 u \partial_{x}-4 u_{x} \\
& \neq \mathcal{D}_{s k-3}
\end{aligned}
$$

hence $\mathcal{D}_{s k-3}$ is not skew-adjoint, let alone can be used to define a Hamiltonian structure for the SK equation. Similarly, we have

$$
\begin{aligned}
\mathcal{D}_{C D G-3}^{*} & =-\partial_{x}^{3}-\partial_{x} \cdot(18 u)-6 u_{x} \\
& =-\partial_{x}^{3}-18 u \partial_{x}-24 u_{x} \\
& \neq-\mathcal{D}_{C D G-3}
\end{aligned}
$$

which shows that it is not skew-adjoint and surely can not be used to construct the Hamiltonian system for the CDG equation. Furthermore, $\mathcal{D}_{\text {Ito-3 }}$ is not skew-adjoint.

On the other hand, we have

$$
\begin{aligned}
\mathcal{D}_{s k-3-1}^{*} & =-\partial_{x}^{3}-\partial_{x} \cdot(4 u)+2 u_{x} \\
& =-\partial_{x}^{3}-4 u \partial_{x}-2 u_{x} \\
& =-\mathcal{D}_{s k-3-1}
\end{aligned}
$$

Therefore $\mathcal{D}_{s k-3-1}$ is a skew-adjoint operator for the SK equation, and plays a suitable candidate for being a Hamiltonian operator, which will be verified later.

In a similar manner, $\mathcal{D}_{\text {Lax }-3}, \mathcal{D}_{K K I-3}, \mathcal{D}_{K K I I-3}$, and $\mathcal{D}_{C D G-3-1}$ are all skewadjoint operators and will be further verified for the Jacobi identity later.

We now turn to the obtained 5-order Lax operator to get

$$
\begin{aligned}
\mathcal{D}_{\text {Lax }-5}^{*}= & {\left[\begin{array}{c}
-\partial_{x}^{5}-\partial_{x}^{3}\left(2 \alpha_{3} u\right)+\left(\alpha_{3}+\beta_{3}\right) u_{x x x} \\
-\partial_{x}\left(\left(3 \alpha_{3}+2 \beta_{3}\right) u_{x x}\right) \\
+\partial_{x x}\left(3 \alpha_{3} u_{x}\right)-\partial_{x}\left(15 u^{2}\right)+15 u u_{x}
\end{array}\right] } \\
= & \left(\begin{array}{c}
-\partial_{x}^{5}-2 \alpha_{3} u_{x x x}-6 \alpha_{3} u_{x x} \partial_{x}-6 \alpha_{3} u_{x} \partial_{x x} \\
-2 \alpha_{3} u \partial_{x x x}+\left(\alpha_{3}+\beta_{3}\right) u_{x x x}-\left(3 \alpha_{3}+2 \beta_{3}\right) u_{x x x} \\
-\left(3 \alpha_{3}+2 \beta_{3}\right) u_{x x} \partial_{x}+3 \alpha_{3} u_{x x x}+6 \alpha_{3} u_{x x} \partial_{x} \\
+3 \alpha_{3} u_{x} \partial_{x x}-15 u u_{x}-15 u^{2} \partial_{x}
\end{array}\right), \\
= & \left(\begin{array}{c}
-\partial_{x}^{5}-2 \alpha_{3} u \partial_{x x x}-\left(\alpha_{3}+\beta_{3}\right) u_{x x x} \\
-\left(3 \alpha_{3}+2 \beta_{3}\right) u_{x x} \partial_{x}-3 \alpha_{3} u_{x} \partial_{x x}-15 u^{2} \partial_{x}-15 u u_{x}
\end{array}\right), \\
= & -\mathcal{D}_{\text {Lax }-5} .
\end{aligned}
$$

Therefore it is skew-adjoint. 
For the 5-order Ito operator, we have

$$
\begin{aligned}
\mathcal{D}_{I t o-5}^{*} & =\left(\begin{array}{c}
-\partial_{x}^{5}-\partial_{x}^{3}\left(2 \alpha_{3} u\right)+\left(\alpha_{3}+\beta_{3}\right) u_{x x x}-\partial_{x}\left(\left(3 \alpha_{3}+2 \beta_{3}\right) u_{x x}\right) \\
+\partial_{x x}\left(3 \alpha_{3} u_{x}\right)-\partial_{x}\left(u^{2}\right)+u u_{x}
\end{array}\right), \\
& =\left(\begin{array}{c}
-\partial_{x}^{5}-2 \alpha_{3} u_{x x x}-6 \alpha_{3} u_{x x} \partial_{x}-6 \alpha_{3} u_{x} \partial_{x x}-2 \alpha_{3} u \partial_{x x x} \\
-\left(\alpha_{3}+\beta_{3}\right) u_{x x x}-\left(3 \alpha_{3}+2 \beta_{3}\right) u_{x x x}-\left(3 \alpha_{3}+2 \beta_{3}\right) u_{x x} \partial_{x} \\
+3 \alpha_{3} u_{x x x}+6 \alpha_{3} u_{x x} \partial_{x}+3 \alpha_{3} u_{x} \partial_{x x}-u^{2} \partial_{x}-u u_{x}
\end{array}\right), \\
& =\left(\begin{array}{c}
-\partial_{x}^{5}-2 \alpha_{3} u \partial_{x x x}-\left(\alpha_{3}+\beta_{3}\right) u_{x x x}-\left(3 \alpha_{3}+2 \beta_{3}\right) u_{x x} \partial_{x} \\
-3 \alpha_{3} u_{x} \partial_{x x}-u^{2} \partial_{x}-u u_{x}
\end{array}\right), \\
& =-\mathcal{D}_{I t o-5}, \quad
\end{aligned}
$$

hence it is also a skew-adjoint operator.

6.2. Hamiltonian and non-Hamiltonian operator. The skew adjoint property of differential operators are easily checked through (16). Jacobi identity is normally easier to check by examining the closure of the corresponding symplectic form. However, most of the operators are highly nontrivial, making it extremely difficult to invert. Thus we will turn to the use of the method of prolongation. We refer the interested readers to $[18,19]$ for details on this method. Here we simply note that if we define a bi-vector as $\Theta_{\mathcal{D}}=\frac{1}{2} \int \theta \wedge \mathcal{D}(\theta) \mathrm{d} x$, then $\mathcal{D}$ would satisfy the Jacobi identity provided

$$
\operatorname{pr} \mathbf{v}_{\mathcal{D} \theta}\left(\Theta_{\mathcal{D}}\right)=0
$$

Here the assumption is that

$$
\theta \neq \theta[u]
$$

and by definition, prolongation acts only on coefficients functionally dependet on $u$.

Now we examine $\mathcal{D}_{\text {Lax }-3}$ in (29), but we first write its bi-vector form as

$$
\Theta_{\mathcal{D}_{\text {Lax }-3}}=\int\left(\frac{1}{2} \theta \wedge \theta_{x x x}+2 u \theta \wedge \theta_{x}+u_{x} \theta \wedge \theta\right) \mathrm{d} x .
$$

Notice that

$$
\operatorname{pr} \mathbf{v}_{\mathcal{D}_{\text {Lax }-3} \theta}(u)=\theta_{x x x}+4 u \theta_{x}+2 u_{x}
$$

so we have

$$
\begin{aligned}
& \operatorname{pr} \mathbf{v}_{\mathcal{D}_{\text {Lax } 3} \theta}\left(\Theta_{\mathcal{D}_{\text {Lax }-3}}\right) \\
= & \operatorname{pr} \mathbf{v}_{\mathcal{D}_{\text {Lax }-3} \theta} \int\left(\frac{1}{2} \theta \wedge \theta_{x x x}+2 u \theta \wedge \theta_{x}+u_{x} \theta \wedge \theta\right) \mathrm{d} x, \\
= & 2 \int\left(\theta_{x x x} \wedge \theta \wedge \theta_{x}+4 u \theta_{x} \wedge \theta \wedge \theta_{x}+2 u_{x} \theta \wedge \theta \wedge \theta_{x}\right) \mathrm{d} x, \\
= & 0 .
\end{aligned}
$$

This shows that $\mathcal{D}_{\text {Lax-3 }}$ is a Hamiltonian operator, and $\mathcal{H}_{\text {Lax-3 }}=\int\left(\frac{1}{2} u u_{x x}+u^{3}\right) \mathrm{d} x$ is the corresponding Hamiltonian. Following similar procedures, $\mathcal{D}_{K K I-3}, \mathcal{D}_{K K I I-3}$, $\mathcal{D}_{s k-3-1}$ and $\mathcal{D}_{C D G-3-1}$ are all shown to be Hamiltonian operators. 
Furthermore, $\mathcal{D}_{\text {Lax }-5}$ in (52) has the bi-vector form as

$$
\begin{aligned}
\Theta_{\mathcal{D}_{\text {Lax }} 5} & =\frac{1}{2} \int \theta \wedge \mathcal{D}_{\text {Lax }-5}(\theta) \mathrm{d} x \\
& =\frac{1}{2} \int\left(\begin{array}{c}
\theta \wedge \theta_{x x x x x}+2 \alpha_{3} u \theta \wedge \theta_{x x x}+\left(\alpha_{3}+\beta_{3}\right) \theta \wedge u_{x x x} \theta \\
+\left(3 \alpha_{3}+2 \beta_{3}\right) \theta \wedge u_{x x} \theta_{x}+3 \alpha_{3} \theta \wedge u_{x} \theta_{x x} \\
+15 u^{2} \theta \wedge \theta_{x}+15 \theta \wedge u u_{x} \theta
\end{array}\right) \mathrm{d} x .
\end{aligned}
$$

Notice that

$$
\operatorname{pr} \mathbf{v}_{\mathcal{D}_{\text {Lax }-5} \theta}(u)=\left(\begin{array}{c}
\theta_{x x x x x}+2 \alpha_{3} u \theta_{x x x}+\left(\alpha_{3}+\beta_{3}\right) u_{x x x} \theta \\
+\left(3 \alpha_{3}+2 \beta_{3}\right) u_{x x} \theta_{x}+3 \alpha_{3} u_{x} \theta_{x x} \\
+15 u^{2} \theta_{x}+15 u u_{x} \theta
\end{array}\right),
$$

we have, by using the integration by parts and boundary conditions,

$$
\begin{aligned}
& \operatorname{pr} \mathbf{v}_{\mathcal{D}_{\text {Lax }-5} \theta}\left(\Theta_{\mathcal{D}_{\text {Lax }-5}}\right) \\
& =\frac{1}{2} \int\left[\begin{array}{c}
\left(2 \alpha_{3}+2 \beta_{3}\right)\left(\begin{array}{c}
\theta_{x x x x x}+2 \alpha_{3} u \theta_{x x x}+\left(\alpha_{3}+\beta_{3}\right) u_{x x x} \theta \\
+\left(3 \alpha_{3}+2 \beta_{3}\right) u_{x x} \theta_{x}+3 \alpha_{3} u_{x} \theta_{x x} \\
+15 u^{2} \theta_{x}+15 u u_{x} \theta
\end{array}\right) \wedge \theta \wedge \theta_{x x x} \\
-2 \beta_{3}\left(\begin{array}{c}
\theta_{x x x x x}+2 \alpha_{3} u \theta_{x x x}+\left(\alpha_{3}+\beta_{3}\right) u_{x x x} \theta \\
+\left(3 \alpha_{3}+2 \beta_{3}\right) u_{x x} \theta_{x}+3 \alpha_{3} u_{x} \theta_{x x} \\
+15 u^{2} \theta_{x}+15 u u_{x} \theta
\end{array}\right) \wedge \theta_{x x} \wedge \theta_{x} \\
+30 u\left(\begin{array}{c}
\theta_{x x x x x}+2 \alpha_{3} u \theta_{x x x}+\left(\alpha_{3}+\beta_{3}\right) u_{x x x} \theta \\
+\left(3 \alpha_{3}+2 \beta_{3}\right) u_{x x} \theta_{x}+3 \alpha_{3} u_{x} \theta_{x x} \\
+15 u^{2} \theta_{x}+15 u u_{x} \theta
\end{array}\right) \wedge \theta \wedge \theta_{x}
\end{array}\right] \mathrm{d} x, \\
& =\frac{1}{2} \int\left[\begin{array}{c}
-\left(2 \alpha_{3}+4 \beta_{3}\right) \theta_{x} \wedge \theta_{x x x x} \wedge \theta_{x x x} \\
3 \alpha_{3} u_{x}\left(2 \alpha_{3}+2 \beta_{3}\right) \theta_{x x} \wedge \theta \wedge \theta_{x x x} \\
\left(-4 \alpha_{3} \beta_{3} u-30 u\right) \theta_{x x x} \wedge \theta_{x x} \wedge \theta_{x} \\
-2 \beta_{3}\left(\begin{array}{c}
\left(\alpha_{3}+\beta_{3}\right) u_{x x x} \\
+15 u u_{x}-90 \alpha_{3} u u_{x}
\end{array}\right) \theta \wedge \theta_{x x} \wedge \theta_{x}
\end{array}\right] \mathrm{d} x .
\end{aligned}
$$

When $3 \alpha_{3}+\beta_{3}=10$, we have

$$
\begin{aligned}
& \operatorname{pr} \mathbf{v}_{\mathcal{D}_{\text {Lax }-5} \theta}\left(\Theta_{\mathcal{D}_{\text {Lax }}-5}\right) \\
& =\frac{1}{2} \int\left[\begin{array}{c}
-\left(40-10 \alpha_{3}\right) \theta_{x} \wedge \theta_{x x x x} \wedge \theta_{x x x} \\
3 \alpha_{3} u_{x}\left(20-4 \alpha_{3}\right) \theta_{x x} \wedge \theta \wedge \theta_{x x x} \\
\left(-4 \alpha_{3}\left(10-3 \alpha_{3}\right)-30\right) u \theta_{x x x} \wedge \theta_{x x} \wedge \theta_{x} \\
-2 \beta_{3}\left(\begin{array}{c}
\left(10-2 \alpha_{3}\right) u_{x x x} \\
+\left(15-90 \alpha_{3}\right) u u_{x}
\end{array}\right) \theta \wedge \theta_{x x} \wedge \theta_{x}
\end{array}\right] \mathrm{d} x .
\end{aligned}
$$

Therefore

$$
\operatorname{pr} \mathbf{v}_{\mathcal{D}_{\text {Lax }-5} \theta}\left(\Theta_{\mathcal{D}_{\text {Lax }-5}}\right) \neq 0,
$$

which proves that $\mathcal{D}_{\text {Lax }-5}$ is not Hamiltonian as a form of (49).

To verify the 5-order Ito operator in (54), we first write its bi-vector form as

$$
\begin{aligned}
\Theta_{\mathcal{D}_{\text {Ito }-5}} & =\frac{1}{2} \int \theta \wedge \mathcal{D}_{\text {Ito-5 }}(\theta) \mathrm{d} x \\
= & \frac{1}{2} \int\left[\begin{array}{c}
\theta \wedge \theta_{x x x x x}+2 \alpha_{3} u \theta \wedge \theta_{x x x}+\theta \wedge\left(\alpha_{3}+\beta_{3}\right) u_{x x x} \theta \\
+\theta \wedge\left(3 \alpha_{3}+2 \beta_{3}\right) u_{x x} \theta_{x}+\theta \wedge 3 \alpha_{3} u_{x} \theta_{x x} \\
+\theta \wedge u^{2} \theta_{x}+\theta \wedge u u_{x} \theta
\end{array}\right] \mathrm{d} x .
\end{aligned}
$$


Since

$$
\operatorname{pr} \mathbf{v}_{\mathcal{D}_{\text {Ito- }-5} \theta}(u)=\left(\begin{array}{c}
\theta_{x x x x x}+2 \alpha_{3} u \theta_{x x x}+\left(\alpha_{3}+\beta_{3}\right) u_{x x x} \theta \\
+\left(3 \alpha_{3}+2 \beta_{3}\right) u_{x x} \theta_{x}+3 \alpha_{3} u_{x} \theta_{x x}+u^{2} \theta_{x}+u u_{x} \theta
\end{array}\right),
$$

it is tedious but straightforward to show that

$$
\begin{aligned}
& \operatorname{pr} \mathbf{v}_{\mathcal{D}_{\text {Ito }-5} \theta}\left(\Theta_{\mathcal{D}_{\text {Ito }-5}}\right) \\
& =\frac{1}{2} \int\left[\begin{array}{c}
\left(2 \alpha_{3}+2 \beta_{3}\right) \theta_{x x x x x} \wedge \theta \wedge \theta_{x x x} \\
+3 \alpha_{3}\left(2 \alpha_{3}+2 \beta_{3}\right) u_{x} \theta_{x x} \wedge \theta \wedge \theta_{x x x} \\
-2 \beta_{3}\left(\begin{array}{c}
\theta_{x x x x x}+2 \alpha_{3} u \theta_{x x x} \\
+\left(\alpha_{3}+\beta_{3}\right) u_{x x x} \theta \\
+u u_{x} \theta
\end{array}\right) \theta_{x x} \wedge \theta_{x} \\
+2 u\left(\begin{array}{c}
\theta_{x x x x x}+2 \alpha_{3} u \theta_{x x x} \\
+3 \alpha_{3} u_{x} \theta_{x x}
\end{array}\right) \theta \wedge \theta_{x}
\end{array}\right] \mathrm{d} x, \\
& =\frac{1}{2} \int\left[\begin{array}{c}
-\left(2 \alpha_{3}+2 \beta_{3}\right) \theta_{x x} \wedge \theta_{x x x x} \wedge \theta_{x x} \\
\left(2 \alpha_{3}+4 \beta_{3}\right) \theta_{x x x x x} \wedge \theta_{x} \wedge \theta_{x x} \\
+\left(3 \alpha_{3}\left(2 \alpha_{3}+2 \beta_{3}\right) u_{x}\right) \theta_{x x} \wedge \theta \wedge \theta_{x x x} \\
\left(-4 \alpha_{3} \beta_{3} u-2 u\right) \theta_{x x x} \wedge \theta_{x x} \wedge \theta_{x} \\
\left(\begin{array}{c}
6 \alpha_{3} u u_{x}+2 \beta_{3}\left(\alpha_{3}+\beta_{3}\right) u_{x x x} \\
+2 \beta_{3} u u_{x}
\end{array}\right) \theta_{x x} \wedge \theta \wedge \theta_{x}
\end{array}\right] \mathrm{d} x,
\end{aligned}
$$

by using the integration by parts and boundary conditions.

Notice that with the condition $3 \alpha_{3}+\beta_{3}=3$, the above expression turns out to be

$$
\begin{aligned}
& \operatorname{pr} \mathbf{v}_{\mathcal{D}_{\text {Ito }-5} \theta}\left(\Theta_{\left.\mathcal{D}_{\text {Ito-5 }}\right)}\right) \\
= & \frac{1}{2} \int\left[\begin{array}{c}
-\left(6-4 \alpha_{3}\right) \theta_{x x} \wedge \theta_{x x x x} \wedge \theta_{x x} \\
\left(12-10 \alpha_{3}\right) \theta_{x x x x x} \wedge \theta_{x} \wedge \theta_{x x} \\
+\left(3 \alpha_{3}\left(6-4 \alpha_{3}\right) u_{x}\right) \theta_{x x} \wedge \theta \wedge \theta_{x x x} \\
\left(-4 \alpha_{3}\left(3-3 \alpha_{3}\right) u-2 u\right) \theta_{x x x} \wedge \theta_{x x} \wedge \theta_{x} \\
\neq 0,
\end{array}\right.
\end{aligned}
$$

for any $\alpha_{3}$. This shows that the Ito equation has no 5 -order Hamiltonian operator as the form of (49).

7. Conclusions. In this paper, we present all of the differential operators for the fifth-order $\mathrm{KdV}$ equation (1) including the order-3 and order-5 differential operators. All the skew-adjoint and Hamiltonian operators have been presented and identified. We show that there are five order-3 Hamiltonian operators, but no order- 5 Hamiltonian operators existed for the fifth-order KdV equations, although they are perfectly skew-adjoint and there are infinite number of them.

\section{REFERENCES}

[1] A. H. SALAS, Exact solutions for the general fifth KdV equation by the exp function method, Appl. Math. Comp., 205 (2008), pp. 291-297.

[2] A. PARKer, On soliton solutions of the Kaup-Kupershmidt equation. II. 'Anomalous' $N$-soliton solutions, Physica D, 137 (2000), pp. 34-48.

[3] A. M. WAZwaz, Abundant solitons solutions for several forms of the fifth-order KdV equation by using the tanh method, Appl. Math. Comput., 182 (2006), pp. 283-300. 
[4] A. M. WAzwaz, $N$-soliton solutions for the combined $K d V$-CDG equation and the $K d V$-Lax equation, Appl. Math. Comput., 203 (2008), pp. 402-407.

[5] J. Satsuma And D. J. Kaup, A Backlund Transformation for a Higher Order Korteweg-De Vries Equation, J. Phys. Soc. Jpn., 43 (1977), pp. 692-697.

[6] K. Sawada And T. Kortera, A method finding N-soliton solutions of the $K d V$ equation and KdV-like eqation, Prog. Theor. Phys., 51 (1974), pp. 1355-1367.

[7] P. J. Caudrey, R. K. Dood, And J. D. Gibbon, A new hierarchy of Korteweg-de Vries equations, Proc. R. Roc. Lond. A., 351 (1976), pp. 407-422.

[8] P. D. LAx, Integrals of nonlinear equations of evolution and solitary waves, Commun. Pure Appl. Math., 62 (1968), pp. 467-490.

[9] A. P. Fordy AND D. J. KAUP, Some remarkable nonlinear transformations, Phys. Lett. A, 75 (1980), p. 325.

[10] D. J. KUAP, On the inverse scattering problem for the cubic eigenvalue problems of the class $\Psi_{3 x}+6 Q \Psi_{x}+6 R \Psi=\lambda \Psi$, Stud. Appl. Math., 62 (1980), pp. 189-216.

[11] B. A. Kupershmidt, A super Korteweg-de Vries equation: An integrable system, Phys. Lett. A, 102 (1984), pp. 213-215.

[12] M. Iто, An extension of nonlinear evolution equations of the $K-d V$ ( $m K-d V)$ type to higher orders, J. Phys. Soc. Jpn., 49 (1980), pp. 771-778.

[13] A. PARKer, On soliton solutions of the Kaup-Kupershmidt equation. I. Direct bilinearisation and solitary wave, Physica D, 137 (2000), pp. 25-48.

[14] M. Iто, A reduce program for finding symmetries of nonlinear evolution equations with uniform rank, Comp. Phys. Commun., 42 (1986), pp. 351-357.

[15] I. M. Gelfand And L. A. DikKI, The resolvents and hamiltonian systems, Funkts. Anal. Prilozh., 11 (1977), pp. 11-27.

[16] F. Magri, A simple model of the integrable Hamiltonian system, J. Math. Phys, 19 (1978), pp. $1156-1162$.

[17] C. T. Lee, J. L. Liu, C. C. Lee, And Y. H. Kang, The second-order KdV equation and its soliton-like solution, Modern Physics Letters, Mod. Phys. Lett. B, 23 (2009), pp. 17711780 .

[18] P. J. OLver, Applications of Lie groups to differential equations, 2nd edition, Springer-Verlag, New York, 2000.

[19] C. T. LeE, Multi-soliton solution of the Two-Mode KdV equation, Ph.D. thesis, Oxford Univeristy, UK, 2007. 